\title{
Treatment Adherence and Its Associated Factors in Patients with Type 2 Diabetes: Results from the Rio de Janeiro Type 2 Diabetes Cohort Study
}

\author{
Fernanda S. Marinho, ${ }^{1}$ Camila B. M. Moram, ${ }^{1}$ Priscila C. Rodrigues, ${ }^{1}$ Nathalie C. Leite, ${ }^{2}$ \\ Gil F. Salles, ${ }^{2}$ and Claudia R. L. Cardoso $\mathbb{D D}^{2}$ \\ ${ }^{1}$ Department of Occupational Therapy, University Hospital Clementino Fraga Filho, School of Medicine, \\ Universidade Federal do Rio de Janeiro, Brazil \\ ${ }^{2}$ Department of Internal Medicine, University Hospital Clementino Fraga Filho, School of Medicine, \\ Universidade Federal do Rio de Janeiro, Brazil
}

Correspondence should be addressed to Claudia R. L. Cardoso; claudiacardoso@hucff.ufrj.br

Received 17 July 2018; Accepted 22 October 2018; Published 27 November 2018

Academic Editor: Eusebio Chiefari

Copyright (c) 2018 Fernanda S. Marinho et al. This is an open access article distributed under the Creative Commons Attribution License, which permits unrestricted use, distribution, and reproduction in any medium, provided the original work is properly cited.

\begin{abstract}
Objectives. To investigate treatment adherence in patients with type 2 diabetes and to evaluate its associated factors. Methods. The Summary of Diabetes Self-Care Activities (SDSCA) questionnaire was used to assess treatment adherence. Good adherence was defined as $\geq 5$ days a week in each SDSCA item. Pain, emotional, and physical domains of the SF-36 quality of life questionnaire and the Canadian Occupational Performance Measure (COPM) were also evaluated. Multivariable logistic regressions explored the independent correlates of good general adherence and of specific items of the SDSCA (diet, exercise, and medications). Results. Good adherence was $93.5 \%$ for medication use, 59.3\% for foot care, 56.1\% for blood glucose monitoring, $29.2 \%$ for diet, and $22.5 \%$ for exercise. Patients with general good adherence had lower BMI, better serum lipid profile, higher values of functional capacity, emotional and pain domains of SF-36, better occupational performance, and lower prevalence of pain or limitation in the upper and lower limbs than patients with worse adherence. The variables associated with good adherence were younger age, lower BMI, presence of macrovascular complications, better occupational performance and emotional domain of SF-36, and higher HDL cholesterol levels. The presence of pain/limitation in the upper limbs was associated with worse adherence. Good medication adherence was associated with longer diabetes duration, lower BMI, and lower $\mathrm{HbA}_{1 \mathrm{c}}$ levels. Higher values of pain and emotional domains of the SF-36 and lower BMI were related to better exercise and diet adherence, while the presence of peripheral neuropathy and joint pain/limitation were associated with worse exercise adherence. Conclusions. Emotional and physical performances are important determinants of good diabetic treatment adherence. Good adherence has beneficial impact on BMI, lipid, and glycemic control.
\end{abstract}

\section{Introduction}

Type 2 diabetes prevalence is progressively growing worldwide due to increases in population ageing and obesity. Around 451 million adults are estimated to have diabetes, most of them living in low- and middle-income countries. If these trends continue, by year 2045, 693 million people will have diabetes [1]. Chronic diabetic complications lead to increased morbidity and precocious mortality due to inadequately controlled diabetes, causing an important burden to individuals, families, society, and health care systems worldwide [2].

Subjects with diabetes need to perform self-care activities to prevent short- and long-term complications related to inadequate disease control and to improve their quality of life. Self-care is considered the keystone of diabetes treatment. Hence, to evaluate the proper adherence to diabetes self-care is fundamental to identify and understand the 
trouble areas in diabetes management. This may help to improve diabetes control and to decrease the burden of complications. Among the questionnaires used to evaluate diabetes self-care, the most commonly employed is the Summary of Diabetes Self-Care Activities (SDSCA). This instrument has been evaluated in many studies and demonstrated satisfactory psychometric properties [3].

It has been suggested that psychosocial factors, such as depression and emotional stress [4-6], as well as the development of chronic degenerative complications, particularly peripheral neuropathy, are associated with worsening of general health condition [7]. Furthermore, the presence of functional disability, defined as difficulty in executing daily life activities and the tasks necessary for the independent functioning in daily life instrumental activities, may also impact on diabetes self-care $[8,9]$. Functional disability seems to be more frequent in older adults with diabetes than in those without diabetes [9-11]. The most commonly used strategy to evaluate disabilities is based on self-reported levels of difficulty in performing mobility tasks, in instrumental activities of daily living and in basic activities of daily living. The Canadian Occupational Performance Measure (COPM) is an outcome measure designed to aid patients to recognize, prioritize, and evaluate the most important disabilities they find in their occupational performance [12]. All these factors, in conjunction, may lead to a decrease in adherence to diabetes self-care daily activities. Hence, to identify the factors associated with better or worse adherence to diabetes self-care is potentially important to achieve future better outcomes in type 2 diabetes management.

Therefore, we intended to investigate the self-care behavior of middle-aged to elderly type 2 diabetes individuals, using the SDSCA questionnaire, and the factors associated with good adherence to self-care recommendations. In particular, we investigate the associations with the presence of chronic degenerative complications, with emotional, physical, and pain domains of life quality evaluated by the Medical Outcomes Study 36-Item Short-Form Health Survey (SF-36) questionnaire, with the profile of disabilities evaluated by the COPM and, additionally, with parameters of diabetes control.

\section{Methods}

This was a cross-sectional study nested within the Rio de Janeiro Type 2 Diabetes Cohort Study, with 476 type 2 diabetic patients in regular attendance in the outpatient clinic of a tertiary care university hospital. The specific exclusion criteria of this study were patients with difficulties to understand the questionnaire due to cognitive problems and patients who did not agree or could not participate in the study. All participants gave written informed consent, and the local Ethics Committee had previously approved the study protocol. The characteristics of this cohort, the baseline procedures, and the diagnostic definitions have been detailed elsewhere [13-15]. All patients were submitted to a standard protocol at a study entry that included a complete clinical examination and laboratory evaluation [13-15]. Pain or joint limitations, which hindered the performance of some daily activities, were investigated by a standard questionnaire that included inquiries on the spine and lower and upper limbs. The SF-36 questionnaire was used to evaluate pain and emotional and physical functional domains of life quality, which has been translated and validated in Brazil [16]. Higher values on SF-36 mean better quality of life in these domains. Subjects were also interviewed to identify the activities that they presented greater difficulty to perform by the Canadian Occupational Performance Measure (COPM), as previously described [17]. Higher values in the COPM mean better occupational performance.

\subsection{Summary of Diabetes Self-Care Activities (SDSCA)} Questionnaire. The revised Summary of Diabetes Self-Care Activities (SDSCA) questionnaire was employed, translated to Portuguese, and previously validated to the Brazilian population [18]. The evaluation was performed from February 2012 to February 2013, by individual interviews conducted by a single experienced occupational therapist. The SDSCA questionnaire evaluates the performance of activities by the patients and their compliance with prescription and other health care professional recommendations. It assesses aspects of the treatment regimen of diabetes over the previous seven days, grouped into six self-care dimensions: diet care (general and specific), physical activity, use of medication, blood glucose monitoring (evaluated only on the 311 patients using insulin), foot care, and smoking. Response options range from 0 to 7 to match the number of days of the week. The higher the number of days per week performing that activity, the higher the level of adherence to this recommendation. The questionnaire also evaluates the average number of cigarettes smoked per day. Patients were considered having general good adherence to diabetic treatment if they were not current smokers and reported at least 5 days per week of compliance to diet, exercise, foot care, and medication use. However, for the questions of eating high fat foods and meals including sweets (specific diet), it was considered good adherence $\leq 2$ days per week. The 5 -day criterion was also used for the other separate item of the SDSCA questionnaire.

2.2. Statistical Analysis. Continuous variables were described as the means and SDs when normally distributed and as the medians and interquartile range when asymmetrically distributed. An unpaired $t$-test or Mann-Whitney tests and chi-squared tests, where adequate, were used to compare variables between patients according to general adherence to diabetes treatment. The covariates independently associated with general good adherence and separated for adherence to diet, physical activity, and medication use were assessed by multivariate logistic regressions using a backward stepwise selection procedure, where a $p$ value $<0.10$ was the criterion to remain into the final models. The candidate variables to enter the models, based on biological plausibility, were age, sex, BMI, diabetes duration, SF-36 pain, physical activity and emotional domains, pain or joint limitations on the spine and lower and upper limbs, COPM score (occupational performance), presence of each macrovascular and microvascular complications, clinic blood pressure levels, glycated hemoglobin $\left(\mathrm{HbA}_{1 \mathrm{c}}\right)$, and serum 
lipid levels (LDL- and HDL cholesterol and triglycerides). Age and sex were forced into all models, regardless of their significance. When the COPM score was entered as a candidate variable into the multivariate analysis, the SF-36 physical activity domain was not included. Similarly, when pain or joint limitations on the spine and lower and upper limbs were entered into the multivariate analysis, the SF-36 pain domain was not included. Results were presented as the odds ratios with their respective $95 \%$ confidence intervals. All statistics were performed with the SPSS statistical package version 19.0 (SPSS Inc., Chicago, IL, USA), and a two-tailed $p$ value $<0.05$ was regarded significant.

\section{Results}

3.1. The Summary of Diabetes Self-Care Activities. Table 1 presents the results of specific items of the SDSCA questionnaire, and Figure 1 outlines the proportion of patients considered adherent ( 5 or more days in the last week) for each SDSCA item. In general, patients had a poor adherence to diet and exercise, a moderate adherence to regular blood sugar assessments and foot care, and a good adherence to medications and not smoking. Overall, only $20 \%$ of the patients were considered with good general adherence to diabetes treatment.

3.2. Characteristics of All Patients and according to General Adherence. Table 2 presents data of all patients and of those considered overall adherent ( $\geq 5$ days a week) and nonadherent ( $<5$ days a week) by the self-reported SDSCA questionnaire. Patients with better adherence had a lower BMI and a better serum lipid profile, particularly higher HDL cholesterol and lower triglycerides levels, than nonadherent patients. They also had better physical, functional, emotional, and pain domains of the SF-36 questionnaire; better occupational performance evaluated by $\mathrm{CPOM}$; and lower prevalences of limitation or pain in the upper and lower limbs than those patients with worse general adherence.

\subsection{Variables Associated with General Adherence and with} Specific Items of Diet, Exercise, and Medication Use of the SDSCA Questionnaire. Results of the multivariate logistic regression analyses of the independent correlates of optimal general adherence and of the specific correlates of diet, exercise, and medication adherence are presented in Table 3. Younger age, lower BMI, higher HDL cholesterol levels, and the presence of macrovascular complications were related to better general adherence. Higher values of the SF-36 emotional domain and better occupational performance were also associated with better general adherence, while the presence of limitation/pain in the upper limbs was associated with worse general adherence.

Good adherence to diet was associated with higher values of HDL cholesterol, better occupational performance measured by CPOM, and better pain domain of the SF-36 questionnaire, while the presence of limitation/pain in the upper limbs was associated with worse adherence to diet. Regarding adherence to regular exercise, better pain and emotional domains on the SF-36 questionnaire, lower BMI, and better
TABLE 1: Results of the Summary of Diabetes Self-Care Activities (SDSCA) questionnaire in 476 type 2 diabetic patients.

\begin{tabular}{|c|c|}
\hline Self-care activities & $\begin{array}{c}\text { Number of } \\
\text { days* }\end{array}$ \\
\hline \multicolumn{2}{|l|}{ General diet questions } \\
\hline Follow a healthful eating plan in the last week & $0(0-6)$ \\
\hline $\begin{array}{l}\text { Follow an eating plan (on average per week, } \\
\text { over the past month) }\end{array}$ & $0(0-6)$ \\
\hline \multicolumn{2}{|l|}{ Specific diet questions } \\
\hline $\begin{array}{l}\text { Eat five or more servings of fruits and vegetables } \\
\text { in the last week }\end{array}$ & $0(0-7)$ \\
\hline Eat high-fat foods in the last week & $4(2-7)$ \\
\hline Eat sweets in the last week & $1(0-2)$ \\
\hline \multicolumn{2}{|l|}{ Exercise } \\
\hline $\begin{array}{l}\text { Participate in at least } 30 \text { minutes of physical } \\
\text { activity, in the last week }\end{array}$ & $7(3-7)$ \\
\hline $\begin{array}{l}\text { Participate in a specific exercise session in the } \\
\text { last week }\end{array}$ & $0(0-2)$ \\
\hline \multicolumn{2}{|l|}{ Blood sugar testing (patients using insulin $=311$ ) } \\
\hline Test blood sugar in the last week & $2(0-7)$ \\
\hline $\begin{array}{l}\text { Test blood sugar according to the number of } \\
\text { times recommended by your health care } \\
\text { provider in the last week }\end{array}$ & $5.5(0-7)$ \\
\hline \multicolumn{2}{|l|}{ Foot care } \\
\hline Check feet in the last week & $7(1-7)$ \\
\hline Inspect the inside of shoes in the last week & $7(0-7)$ \\
\hline Dry between toes after washing, in the last week & $7(7-7)$ \\
\hline \multicolumn{2}{|l|}{ Medications } \\
\hline $\begin{array}{l}\text { Take recommended diabetic medications in } \\
\text { the last week }\end{array}$ & $7(7-7)$ \\
\hline $\begin{array}{l}\text { Take recommended insulin injections in the } \\
\text { last week }\end{array}$ & $7(7-7)$ \\
\hline $\begin{array}{l}\text { Take recommended number of diabetes pills } \\
\text { in the last week }\end{array}$ & $7(7-7)$ \\
\hline \multicolumn{2}{|l|}{ Smoking } \\
\hline Patients who smoked during the past 7 days (\%) & 6.1 \\
\hline Number of cigarettes smoked in a day & $13(5-15)$ \\
\hline Never smokers (\%) & 51.5 \\
\hline Past smokers (\%) & 42.4 \\
\hline
\end{tabular}

*Values are the median number of days and interquartile range, except for smoking status, which are proportions and median number of cigarettes (for current smokers).

occupational performance were independently associated with good adherence, while the presence of diabetic peripheral neuropathy and pain/limitation in the spine were associated with poor adherence to exercise. Better self-reported adherence to medication use was independently associated with lower $\mathrm{HbA}_{1 \mathrm{c}}$ levels, longer diabetes duration, and lower BMI.

\section{Discussion}

This study has three main findings. First, it demonstrated that adherence to self-care recommendations, evaluated by the SDSCA questionnaire, in middle-aged to elderly type 


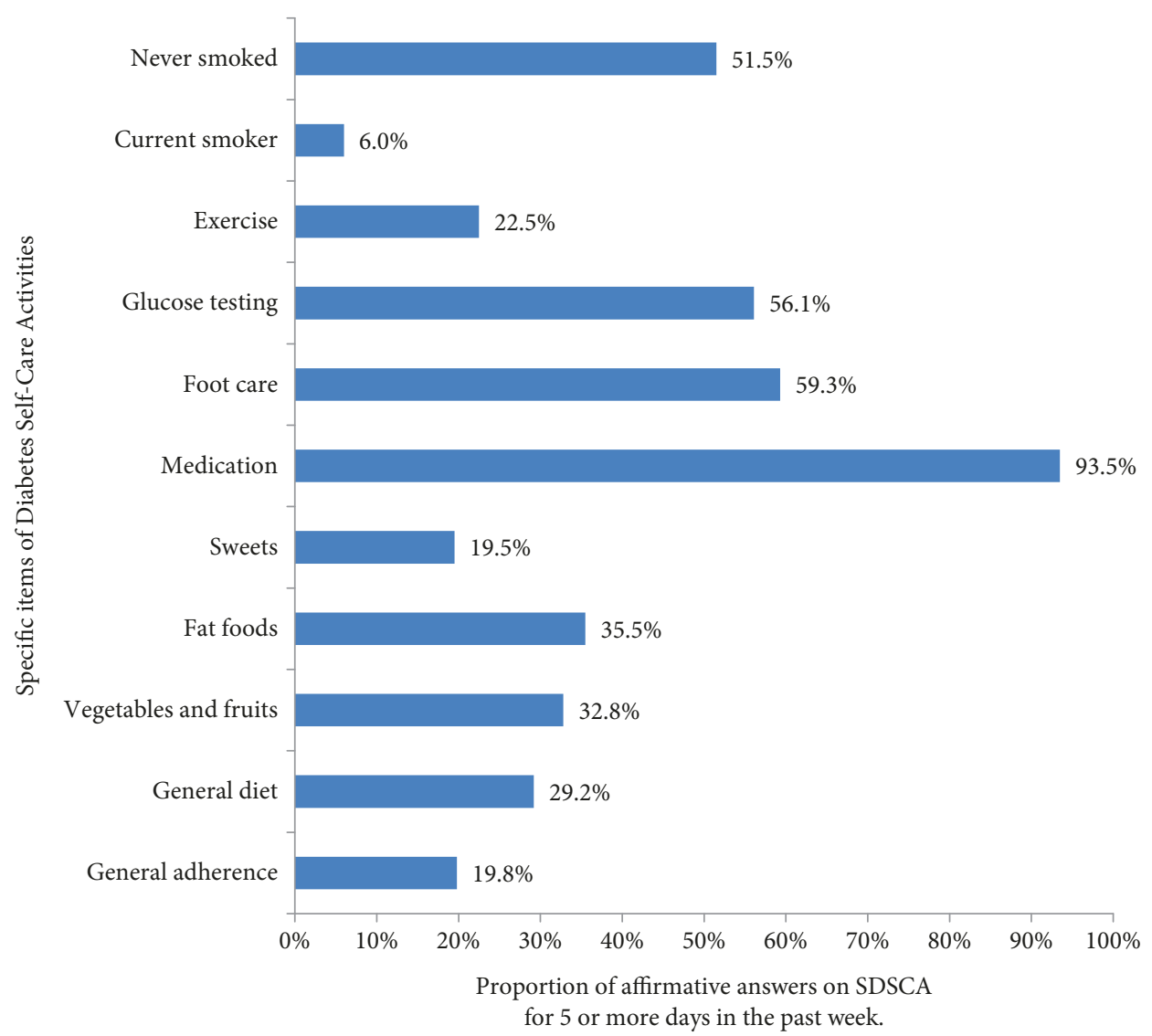

FIGURE 1: Summary of Diabetes Self-Care Activities and percentage of affirmative answers to SDSCA of 5 days or more, except for sweets and fat food that were considered affirmative answers to two days or less.

2 diabetes was good (>90\%) only for medication use and nonsmoking. It was moderate for foot care (59\%) and for blood glucose monitoring (56\%) and was particularly poor $(<30 \%)$ for diet and physical activity. Second, and the most important, better general adherence was associated with younger age, lower BMI, better occupational performance (reflected by higher values of the COPM, which is a measure of self-reported disabilities), better emotional domain of the SF-36 quality of life questionnaire, and higher values of HDL cholesterol. The presence of limitation/pain in the upper limbs was associated with worse general adherence. Third, good self-reported adherence to medication use was associated with better glycemic control, good adherence to diet was associated with better serum lipid profile, and a lower BMI was associated with almost all items of self-care activities, suggesting the importance of treatment adherence to better overall diabetes control. Furthermore, better scores of pain and emotional domains of the SF-36 questionnaire, better occupational performance, and absence of joint pain/limitation and of diabetic peripheral neuropathy were also associated with good adherence to diet and physical activity, reflecting the importance of physical and emotional aspects to better treatment adherence. These findings suggest that future studies with interventions to improve functional disabilities, especially by reducing pain in the upper limbs and spine and preventing peripheral neuropathy progression, and emotional support with treatment of depression/anxiety may help to improve overall adherence to diabetes treatment with consequent impact on better outcomes.

In this study, diabetes medication adherence was associated with lower $\mathrm{HbA}_{1 \mathrm{c}}$ levels. The relation between medication adherence and glucose control is still subject to debate, in which most studies showed significant associations between self-reported medication adherence and $\mathrm{HbA}_{1 \mathrm{c}}$, using different instruments [19-22], while others did not demonstrate associations between self-reported adherence and $\mathrm{HbA}_{1 \mathrm{c}}$ [23]. Nonetheless, associations with other measures of adherence and $\mathrm{HbA}_{1 \mathrm{c}}$ may be modest, or not present, because glycemic control is dependent of various factors, besides medication adherence, including diet and exercise, grade of insulin deficit, and adequacy of medication in use $[24,25]$. Further, a meta-analysis [26] in type 1 diabetes investigating the relationship between treatment adherence and $\mathrm{HbA}_{1 \mathrm{c}}$ showed that adherence explained a small part of $\mathrm{HbA}_{1 \mathrm{c}}$ variation $(<8 \%)$. It also demonstrated no difference in the strength of associations in studies that used self-reported or objectively monitored medication adherence [26]. Likewise, a study in type 2 diabetes assessing relations between medication adherence and $\mathrm{HbA}_{1 \mathrm{c}}$ demonstrated that adherence explained only $4 \%$ of baseline $\mathrm{HbA}_{1 \mathrm{c}}$ and $1.7 \%$ of $\mathrm{HbA}_{1 \mathrm{c}}$ change [27]. So validating self-reported 
TABLE 2: Characteristics of all patients and according to general adherence (5 or more days per week in all items of the SDSCA questionnaire, except in exercise).

\begin{tabular}{|c|c|c|c|c|}
\hline Characteristics & $\begin{array}{l}\text { All patients } \\
(n=476)\end{array}$ & $\begin{array}{l}\text { Adherent patients } \\
\quad(n=94)\end{array}$ & $\begin{array}{l}\text { Nonadherent patients } \\
(n=382)\end{array}$ & $p$ value \\
\hline Age (years) & $65(10.7)$ & $64(11.7)$ & $65(10.5)$ & 0.29 \\
\hline Diabetes duration (years) & $8(3-15)$ & $8(3-15)$ & $8(3-15)$ & 0.94 \\
\hline Gender, female (\%) & 63.9 & 63.6 & 64.9 & 0.82 \\
\hline Schooling years, $\geq 8$ years (\%) & 25.5 & 24.9 & 27.5 & 0.59 \\
\hline Social security situation (\% of retirement) & 38 & 43.6 & 36.6 & 0.69 \\
\hline Marital status (\% of married) & 64.1 & 70.1 & 62.6 & 0.48 \\
\hline Body mass index $\left(\mathrm{kg} / \mathrm{m}^{2}\right)$ & $29.8(4.7)$ & $28.7(4.1)$ & $30.1(4.8)$ & 0.005 \\
\hline \multicolumn{5}{|l|}{ Diabetes treatment (\%) } \\
\hline Metformin & 86.8 & 86.6 & 86.9 & 0.95 \\
\hline Sulfonylureas & 20.8 & 21.5 & 20.6 & 0.86 \\
\hline Insulin & 68.4 & 67.7 & 68.5 & 0.89 \\
\hline \multicolumn{5}{|l|}{ Chronic diabetic complications (\%) } \\
\hline Retinopathy & 31.0 & 34.8 & 30.1 & 0.38 \\
\hline Nephropathy & 28.2 & 28.0 & 28.2 & 0.96 \\
\hline Peripheral neuropathy & 27.4 & 24.5 & 28.2 & 0.47 \\
\hline Cerebrovascular disease & 6.5 & 7.4 & 6.3 & 0.68 \\
\hline Coronary artery disease & 14.7 & 16.0 & 14.4 & 0.70 \\
\hline Peripheral arterial disease & 13.5 & 12.6 & 17.0 & 0.26 \\
\hline Arterial hypertension (\%) & 85.3 & 81.9 & 86.1 & 0.30 \\
\hline $\mathrm{SBP}(\mathrm{mmHg})$ & $139(18)$ & $139(20)$ & $138(18)$ & 0.81 \\
\hline $\mathrm{DBP}(\mathrm{mmHg})$ & $76(11)$ & $75(11)$ & $76(11)$ & 0.34 \\
\hline Dyslipidemia (\%) & 87.6 & 85.1 & 88.2 & 0.41 \\
\hline \multicolumn{5}{|l|}{ Laboratory variables } \\
\hline \multirow{2}{*}{$\mathrm{HbA}_{1 \mathrm{c}}(\%)(\mathrm{mmol} / \mathrm{mol})$} & $7.9(1.6)$ & $7.9(1.6)$ & $7.9(1.7)$ & 0.98 \\
\hline & $63(15.6)$ & $63(15.6)$ & $63(15.7)$ & \\
\hline LDL cholesterol (mmol/L) & $2.46(0.78)$ & $2.45(0.69)$ & $2.46(0.81)$ & 0.92 \\
\hline HDL cholesterol (mmol/L) & $1.19(0.36)$ & $1.27(0.36)$ & $1.17(0.35)$ & 0.007 \\
\hline Triacylglycerol (mmol/L) & $1.46(1.07-2.04)$ & $1.29(0.97-1.73)$ & $1.50(1.11-2.13)$ & 0.003 \\
\hline \multicolumn{5}{|l|}{ Domains of quality of life (SF-36) } \\
\hline Functional capacity & $49(31.5)$ & $56(32.3)$ & $47(31.1)$ & 0.011 \\
\hline Emotional & $58(45.6)$ & $68(43.3)$ & $55(46.0)$ & 0.014 \\
\hline Pain & $51(30.3)$ & $56(30.8)$ & $49(30.1)$ & 0.041 \\
\hline Occupational performance (COPM) & $4.6(1.9)$ & $5.2(2.0)$ & $4.4(1.9)$ & 0.001 \\
\hline Satisfaction in the performance of activities (COPM) & $4.3(2.3)$ & $4.8(2.5)$ & $4.2(2.2)$ & 0.035 \\
\hline \multicolumn{5}{|l|}{ Limitations/pain (\%) } \\
\hline In the upper limb & 52.3 & 37.2 & 56.0 & 0.001 \\
\hline In the lower limb & 60.3 & 47.9 & 63.4 & 0.006 \\
\hline In the spine & 54.8 & 54.3 & 55.0 & 0.90 \\
\hline
\end{tabular}

Values are the proportions, means (standard deviations), or medians (interquartile range). Abbreviations: SBP, systolic blood pressure; DBP, diastolic blood pressure; $\mathrm{HbA}_{1 \mathrm{c}}$, glycated hemoglobin; HDL, high-density lipoprotein; LDL, low-density lipoprotein; COPM, Canadian Occupational Performance Measure.

adherence by using only clinical endpoints such as $\mathrm{HbA}_{1 \mathrm{c}}$ levels may lead to less precise inferences, due to rather modest influence of adherence on this outcome [24]. Additionally, methods for assessing medication adherence and persistence were highly variable and all meta-analyses had a high degree of heterogeneity [28]. Besides, younger age and longer diabetes duration were also independently associated with better medication adherence. These findings are in agreement, in part, with a large report [29] of diabetic patients treated with noninsulin medications where longer disease duration was associated with better adherence. However, different from our study, older age and male gender were also related to better adherence [29].

Depression has been frequently associated with selfreported worse treatment adherence in patients with diabetes $[4,30]$. Similarly, lower scores on the emotional domain 
TABLE 3: Results of multiple logistic regressions for variables associated with general adherence and, for specific items of diet, exercise and medication adherence of the SDSCA questionnaire.

\begin{tabular}{|c|c|c|c|}
\hline Dependent variable/independent covariates & OR & $95 \% \mathrm{CI}$ & $p$ value \\
\hline \multicolumn{4}{|l|}{ General adherence* } \\
\hline HDL cholesterol (10 mg/dL increase) & 1.25 & $1.06-1.47$ & 0.008 \\
\hline SF-36 emotional domain (each 10-point increase) & 1.07 & $1.01-1.12$ & 0.021 \\
\hline BMI $\left(1 \mathrm{~kg} / \mathrm{m}^{2}\right.$ increase $)$ & 0.94 & $0.89-0.99$ & 0.021 \\
\hline Age (1 year increase) & 0.98 & $0.96-0.998$ & 0.033 \\
\hline Presence of macrovascular complications (yes/no) & 1.62 & $0.96-2.37$ & 0.073 \\
\hline \multicolumn{4}{|l|}{ General adherence** } \\
\hline Pain/limitation in the upper limbs (yes/no) & 0.49 & $0.30-0.81$ & 0.006 \\
\hline HDL cholesterol (10 mg/dL increase) & 1.25 & $1.06-1.47$ & 0.008 \\
\hline COPM (each 1-point increase) & 1.18 & $1.04-1.35$ & 0.012 \\
\hline Age (1-year increase) & 0.97 & $0.95-0.99$ & 0.014 \\
\hline SF-36 emotional domain (each 10-point increase) & 1.07 & $1.01-1.12$ & 0.021 \\
\hline Presence of macrovascular complications (yes/no) & 1.88 & $1.09-3.23$ & 0.022 \\
\hline BMI $\left(1 \mathrm{~kg} / \mathrm{m}^{2}\right.$ increase $)$ & 0.96 & $0.91-1.01$ & 0.098 \\
\hline \multicolumn{4}{|l|}{ General diet* } \\
\hline HDL cholesterol (10 mg/dL increase) & 1.19 & $1.02-1.37$ & 0.022 \\
\hline SF-36 pain domain (each 10-point increase) & 1.08 & $1.01-1.16$ & 0.033 \\
\hline SF-36 emotional domain (each 10-point increase) & 1.04 & $0.99-1.09$ & 0.076 \\
\hline \multicolumn{4}{|l|}{ General diet** } \\
\hline COPM (each 1-point increase) & 1.22 & $1.09-1.36$ & 0.001 \\
\hline Pain/limitation in the upper limbs (yes/no) & 0.59 & $0.38-0.90$ & 0.015 \\
\hline HDL cholesterol (10 mg/dL increase) & 1.19 & $1.02-1.38$ & 0.025 \\
\hline \multicolumn{4}{|l|}{ Exercise* $^{*}$} \\
\hline SF-36 pain domain (each 10-point increase) & 1.13 & $1.04-1.21$ & 0.002 \\
\hline SF-36 emotional domain (each 10-point increase) & 1.09 & $1.03-1.15$ & 0.003 \\
\hline BMI $\left(1 \mathrm{~kg} / \mathrm{m}^{2}\right.$ increase $)$ & 0.94 & $0.89-0.99$ & 0.011 \\
\hline Presence of peripheral neuropathy (yes/no) & 0.55 & $0.31-0.95$ & 0.032 \\
\hline \multicolumn{4}{|l|}{ Exercise ${ }^{* *}$} \\
\hline SF-36 emotional domain (each 10-point increase) & 1.08 & $1.02-1.14$ & 0.009 \\
\hline BMI $\left(1 \mathrm{~kg} / \mathrm{m}^{2}\right.$ increase $)$ & 0.94 & $0.90-0.99$ & 0.029 \\
\hline Presence of peripheral neuropathy (yes/no) & 0.55 & $0.31-0.95$ & 0.032 \\
\hline Pain/limitation in the spine (yes/no) & 0.61 & $0.39-0.98$ & 0.039 \\
\hline COPM (each 1-point increase) & 1.12 & $0.99-1.27$ & 0.066 \\
\hline Female gender & 1.53 & $0.95-2.45$ & 0.082 \\
\hline \multicolumn{4}{|l|}{ Medication $^{\dagger}$} \\
\hline $\mathrm{HbA}_{1 \mathrm{c}}(1 \%$ increase $)$ & 0.73 & $0.62-0.86$ & $<0.001$ \\
\hline BMI $\left(1 \mathrm{~kg} / \mathrm{m}^{2}\right.$ increase $)$ & 0.92 & $0.86-0.99$ & 0.022 \\
\hline Diabetes duration (1-year increase) & 1.04 & $0.99-1.09$ & 0.059 \\
\hline
\end{tabular}

${ }^{*}$ The candidate variables to enter these models were BMI, diabetes duration, SF-36 pain, physical activity and emotional domains, presence of macrovascular and microvascular complications, blood pressure, glycated hemoglobin, and LDL and HDL cholesterol. ${ }^{* *}$ Pain or joint limitation on the spine and lower and upper limbs and COPM entered the model instead of SF-36 pain and physical domain items. ${ }^{\dagger}$ Both models were identical. Age and gender were forced into all models. Abbreviations: BMI, body mass index; COPM, Canadian Occupational Performance Measure.

of the SF-36 questionnaire, which combines depressive and anxiety complains, were associated with worse general adherence in the present study. Additionally, we observed that pain/limitation in the upper limbs was independently associated with worse general adherence, which is a relevant finding. Although musculoskeletal disorders are sometimes unique or more prevalent in people with diabetes, conditions of the upper limb causing pain, discomfort, and limited movements have in general been underdiagnosed and poorly treated, in comparison to the other 
complications of diabetes [31]. Injury to vessels and nerves, protein glycosylation, and augmented collagen in the skin and musculoskeletal connective tissues are some factors that possibly may contribute to the development of musculoskeletal disorders in diabetic patients [32]. They may lead not only to important disabilities and poor quality of life in diabetic patients but also to poor treatment adherence and worse outcomes.

Not unexpectedly, the presence of peripheral neuropathy was associated with worse physical activity adherence and higher values of pain and emotional domains of SF-36 were related to better adherence. Previous studies reported relationships between the presence of neuropathy and depression $[33,34]$. However, the association between the presence of depression and peripheral neuropathy and reduced treatment adherence is less evidenced in diabetic patients [35].

There are limitations in the present study that need to be acknowledged. First, its cross-sectional design allows no speculations about causality, but only associations between adherence and the factors. More importantly, we investigated self-reported treatment adherence, which may result in biased data. However, even objective measurements of adherence, which have been demonstrated to be comparable to self-reported adherence in their associations with glycemic control, [25] have also their own related measurement error [24]. Furthermore, objective measurements of treatment adherence frequently are not practical in clinical settings and are not able to help caregivers to improve treatment adherence of their patients [24]. Another aspect that restricts performing research on treatment adherence is the absence of a standard definition, which limits the comparability of studies and increases the risk of selective reporting [24]. The team of health care providers is compounded of three medical doctors, two nurses, two occupational therapists, and a nutritionist; although the group is homogeneous regarding commitment and support to patients, we did not evaluate if there were differences in the adherence among them, considering that these factors may have a relation with adherence. Finally, this study was conducted in a tertiary care hospital with a patient population of predominantly middle-aged to elderly individuals; thus, our findings may not be generalizable to younger diabetic patients and to patients followed at primary care centers. On the other hand, we included a relatively large number of type 2 diabetic patients, and its main strength is that our well-documented cohort was allowed to perform a comprehensive analysis of possible factors associated with adherence.

In conclusion, this study demonstrated that general adherence to recommended self-care activities in middleaged to elderly patients with type 2 diabetes was poor, particularly because of poor adherence to diet and exercise, that several emotional and physical factors seemed to affect adherence, and that good adherence was associated with better diabetes control. This study suggests that patient-centered interventions addressing improvements in different areas of performance, including pain management and emotional support, may enable greater patient independence and autonomy and may also improve treatment adherence. Whether such interventions will have impact on better clinical outcomes in patients with type 2 diabetes shall be the focus of future studies.

\section{Data Availability}

The Rio de Janeiro Type 2 Diabetes Cohort Study is an ongoing study, and its dataset is not publicly available due to individual privacy of the participants. However, it may be available from the corresponding author on reasonable request.

\section{Disclosure}

The sponsors had no role in the study design, collection and analysis of data, interpretation of results, or preparation, review, and approval of the manuscript.

\section{Conflicts of Interest}

The authors declare that there is no conflict of interest regarding this manuscript.

\section{Acknowledgments}

This study was supported by grants from the Conselho Nacional de Desenvolvimento Científico e Tecnológico $(\mathrm{CNPq}, \mathrm{Brazil})$ and from the Fundação Carlos Chagas Filho de Amparo à Pesquisa do Estado do Rio de Janeiro (FAPERJ, Brazil).

\section{References}

[1] N. H. Cho, J. E. Shaw, S. Karuranga et al., "IDF Diabetes Atlas: global estimates of diabetes prevalence for 2017 and projections for 2045," Diabetes Research and Clinical Practice, vol. 138, pp. 271-281, 2018.

[2] P. Z. Zimmet, D. J. Magliano, W. H. Herman, and J. E. Shaw, "Diabetes: a 21st century challenge," The Lancet Diabetes and Endocrinology, vol. 2, no. 1, pp. 56-64, 2014.

[3] Y. Lu, J. Xu, W. Zhao, and H. R. Han, "Measuring self-care in persons with type 2 diabetes: a systematic review," Evaluation of the Health Professions, vol. 39, no. 2, pp. 131-184, 2015.

[4] J. S. Gonzalez, M. Peyrot, L. A. McCarl et al., "Depression and diabetes treatment nonadherence: a meta-analysis," Diabetes Care, vol. 31, no. 12, pp. 2398-2403, 2008.

[5] L. Fisher, J. T. Mullan, P. Arean, R. E. Glasgow, D. Hessler, and U. Masharani, "Diabetes distress but not clinical depression or depressive symptoms is associated with glycemic control in both cross-sectional and longitudinal analyses," Diabetes Care, vol. 33, no. 1, pp. 23-28, 2009.

[6] L. Fisher, R. E. Glasgow, and L. A. Strycker, "The relationship between diabetes distress and clinical depression with glycemic control among patients with type 2 diabetes," Diabetes Care, vol. 33, no. 5, pp. 1034-1036, 2010.

[7] J. L. Hudson, C. Bundy, P. A. Coventry, and C. Dickens, "Exploring the relationship between cognitive illness representations and poor emotional health and their combined association with diabetes self-care. A systematic review with meta-analysis," Journal of Psychosomatic Research, vol. 76, no. 4, pp. 265-274, 2014. 
[8] R. F. Palmer, D. V. Espino, J. M. Dergance, J. Becho, and K. Markides, "The role of physical activity and diabetes status as a moderator: functional disability among older Mexican Americans," Age and Ageing, vol. 41, no. 6, pp. 752-758, 2012.

[9] E. W. Gregg, G. L. Beckles, D. F. Williamson et al., "Diabetes and physical disability among older U.S. adults," Diabetes Care, vol. 23, no. 9, pp. 1272-1277, 2000.

[10] B. Ryerson, E. F. Tierney, T. J. Thompson et al., "Excess physical limitations among adults with diabetes in the U.S. population, 1997-1999," Diabetes Care, vol. 26, no. 1, pp. 206-210, 2003.

[11] A. Sinclair, T. Dunning, and L. Rodriguez-Mañas, "Diabetes in older people: new insights and remaining challenges," The Lancet Diabetes and Endocrinology, vol. 3, no. 4, pp. 275285, 2015.

[12] M. Law, S. Baptiste, M. McColl, A. Opzoomer, H. Polatajko, and N. Pollock, "The Canadian occupational performance measure: an outcome measure for occupational therapy," Canadian Journal of Occupational Therapy, vol. 57, no. 2, pp. 82-87, 2016.

[13] G. F. Salles, N. C. Leite, B. B. Pereira, E. M. Nascimento, and C. R. L. Cardoso, "Prognostic impact of clinic and ambulatory blood pressure components in high-risk type 2 diabetic patients: the Rio de Janeiro Type 2 Diabetes Cohort Study," Journal of Hypertension, vol. 31, no. 11, pp. 2176-2186, 2013.

[14] C. R. L. Cardoso, M. T. Ferreira, N. C. Leite, and G. F. Salles, "Prognostic impact of aortic stiffness in high-risk type 2 diabetic patients: the Rio de Janeiro Type 2 Diabetes Cohort Study," Diabetes Care, vol. 36, no. 11, pp. 3772-3778, 2013.

[15] C. R. L. Cardoso, N. C. Leite, M. T. Ferreira, and G. F. Salles, "Prognostic importance of baseline and serial glycated hemoglobin levels in high-risk patients with type 2 diabetes: the Rio de Janeiro Type 2 Diabetes Cohort Study," Acta Diabetologica, vol. 52, no. 1, pp. 21-29, 2015.

[16] R. M. Ciconelli, M. B. Ferraz, W. Santos, I. Meinão, and M. R. Quaresma, "Tradução para a língua portuguesa e validação do questionário genérico de avaliação de qualidade de vida SF-36 (Brasil SF-36)," Revista Brasileira de Reumatologia, vol. 39, no. 3, pp. 143-150, 1999.

[17] F. S. Marinho, C. B. M. Moram, P. C. Rodrigues, A. C. O. B. Franzoi, G. F. Salles, and C. R. L. Cardoso, "Profile of disabilities and their associated factors in patients with type 2 diabetes evaluated by the Canadian occupational performance measure: the Rio De Janeiro Type 2 Diabetes Cohort Study," Disability and Rehabilitation, vol. 38, no. 21, pp. 2095-2101, 2016.

[18] M. J. Michels, M. H. C. Coral, T. M. Sakae, T. B. Damas, and L. M. Furlanetto, "Questionnaire of Diabetes Self-Care Activities: translation, cross-cultural adaptation and evaluation of psychometric properties," Arquivos Brasileiros de Endocrinologia e Metabologia, vol. 54, no. 7, pp. 644-651, 2010.

[19] Y. Wang, J. Lee, M. P. H. S. Toh, W. E. Tang, and Y. Ko, "Validity and reliability of a self-reported measure of medication adherence in patients with type 2 diabetes mellitus in Singapore," Diabetic Medicine, vol. 29, no. 9, pp. e338-e344, 2012.

[20] H. K. Al-Qazaz, M. A. Hassali, A. A. Shafie, S. A. Sulaiman, S. Sundram, and D. E. Morisky, "The eight-item Morisky Medication Adherence Scale MMAS: translation and validation of the Malaysian version," Diabetes Research and Clinical Practice, vol. 90, no. 2, pp. 216-221, 2010.
[21] M. Tiv, J. F. Viel, F. Mauny et al., "Medication adherence in type 2 diabetes: the ENTRED study 2007, a French Population-Based Study," PLoS One, vol. 7, no. 3, article e32412, 2012.

[22] J. E. Aikens and J. D. Piette, "Longitudinal association between medication adherence and glycaemic control in type 2 diabetes," Diabetic Medicine, vol. 30, no. 3, pp. 338-344, 2013.

[23] H. W. Cohen, C. Shmukler, R. Ullman, C. M. Rivera, and E. A. Walker, "Measurements of medication adherence in diabetic patients with poorly controlled $\mathrm{HbA}_{1 \mathrm{c}}$ " Diabetic Medicine, vol. 27, no. 2, pp. 210-216, 2010.

[24] J. S. Gonzalez and H. E. Schneider, "Methodological issues in the assessment of diabetes treatment adherence," Current Diabetes Reports, vol. 11, no. 6, pp. 472-479, 2011.

[25] J. S. Gonzalez, H. E. Schneider, D. J. Wexler et al., "Validity of medication adherence self-reports in adults with type 2 diabetes," Diabetes Care, vol. 36, no. 4, pp. 831-837, 2013.

[26] K. K. Hood, C. M. Peterson, J. M. Rohan, and D. Drotar, "Association between adherence and glycemic control in pediatric type 1 diabetes: a meta-analysis," Pediatrics, vol. 124, no. 6, pp. e1171-e1179, 2009.

[27] J. M. Schectman, M. M. Nadkarni, and J. D. Voss, "The association between diabetes metabolic control and drug adherence in an indigent population," Diabetes Care, vol. 25, no. 6, pp. 1015-1021, 2002.

[28] A. McGovern, Z. Tippu, W. Hinton, N. Munro, M. Whyte, and S. de Lusignan, "Comparison of medication adherence and persistence in type 2 diabetes: a systematic review and metaanalysis," Diabetes, Obesity \& Metabolism, vol. 20, no. 4, pp. 1040-1043, 2018.

[29] M. S. Kirkman, M. T. Rowan-Martin, R. Levin et al., "Determinants of adherence to diabetes medications: findings from a large pharmacy claims database," Diabetes Care, vol. 38, no. 4, pp. 604-609, 2015.

[30] K. Capoccia, P. S. Odegard, and N. Letassy, "Medication adherence with diabetes medication: a systematic review of the literature," The Diabetes Educator, vol. 42, no. 1, pp. 3471, 2016.

[31] M. Merashli, T. A. Chowdhury, and A. S. M. Jawad, "Musculoskeletal manifestations of diabetes mellitus," QJM, vol. 108, no. 11, pp. 853-857, 2015.

[32] R. P. Kim, "The musculoskeletal complications of diabetes," Current Diabetes Reports, vol. 2, no. 1, pp. 49-52, 2002.

[33] K. Ishizawa, T. Babazono, Y. Horiba et al., "The relationship between depressive symptoms and diabetic complications in elderly patients with diabetes: analysis using the Diabetes Study from the Center of Tokyo Women's Medical University (DIACET)," Journal of Diabetes and its Complications, vol. 30, no. 4, pp. 597-602, 2016.

[34] N. Papanas and D. Ziegler, "Risk factors and comorbidities in diabetic neuropathy: an update 2015," The Review of Diabetic Studies, vol. 12, no. 1-2, pp. 48-62, 2015.

[35] B. Timar, R. Timar, A. Schiller et al., "Impact of neuropathy on the adherence to diabetes-related self-care activities: a crosssectional study," Patient Preference and Adherence, vol. 10, pp. 1169-1175, 2016. 


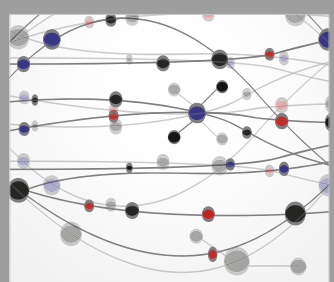

The Scientific World Journal
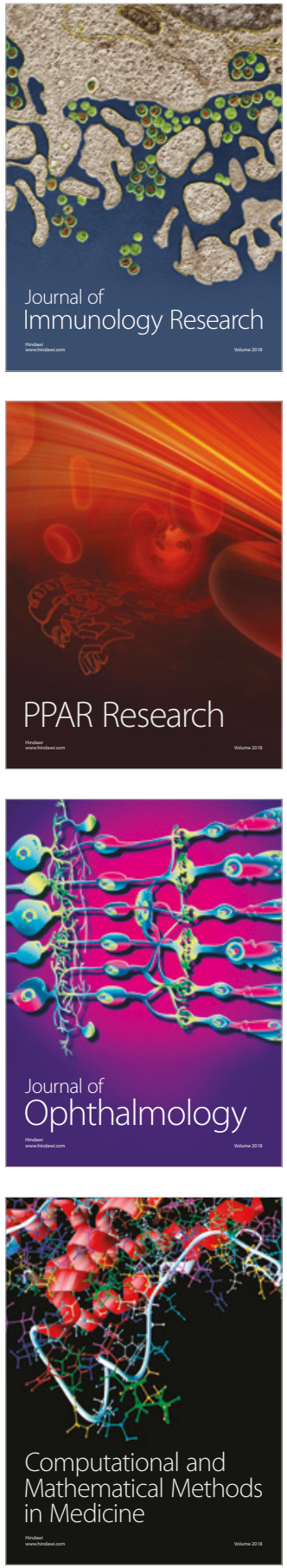

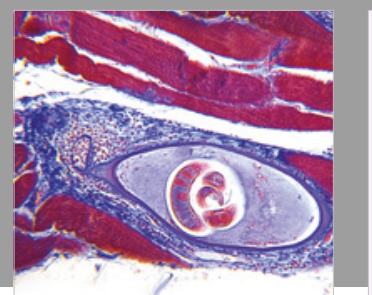

Gastroenterology Research and Practice

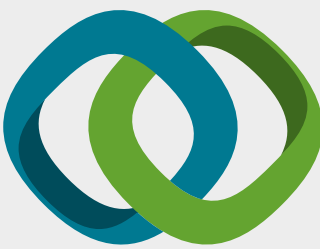

\section{Hindawi}

Submit your manuscripts at

www.hindawi.com
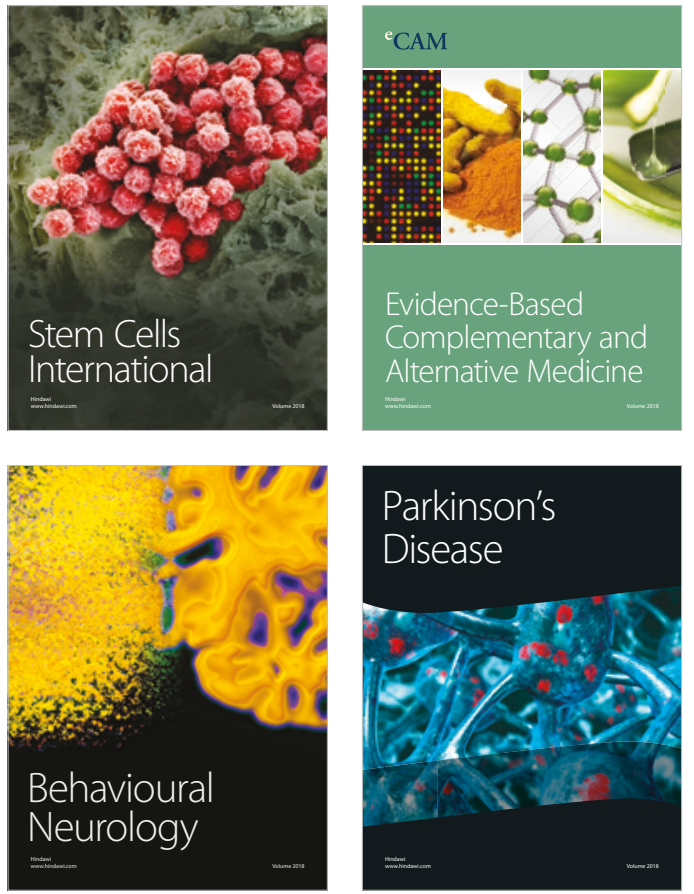

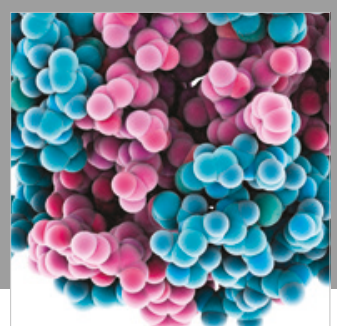

ournal of

Diabetes Research

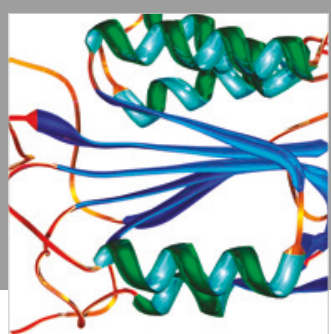

Disease Markers
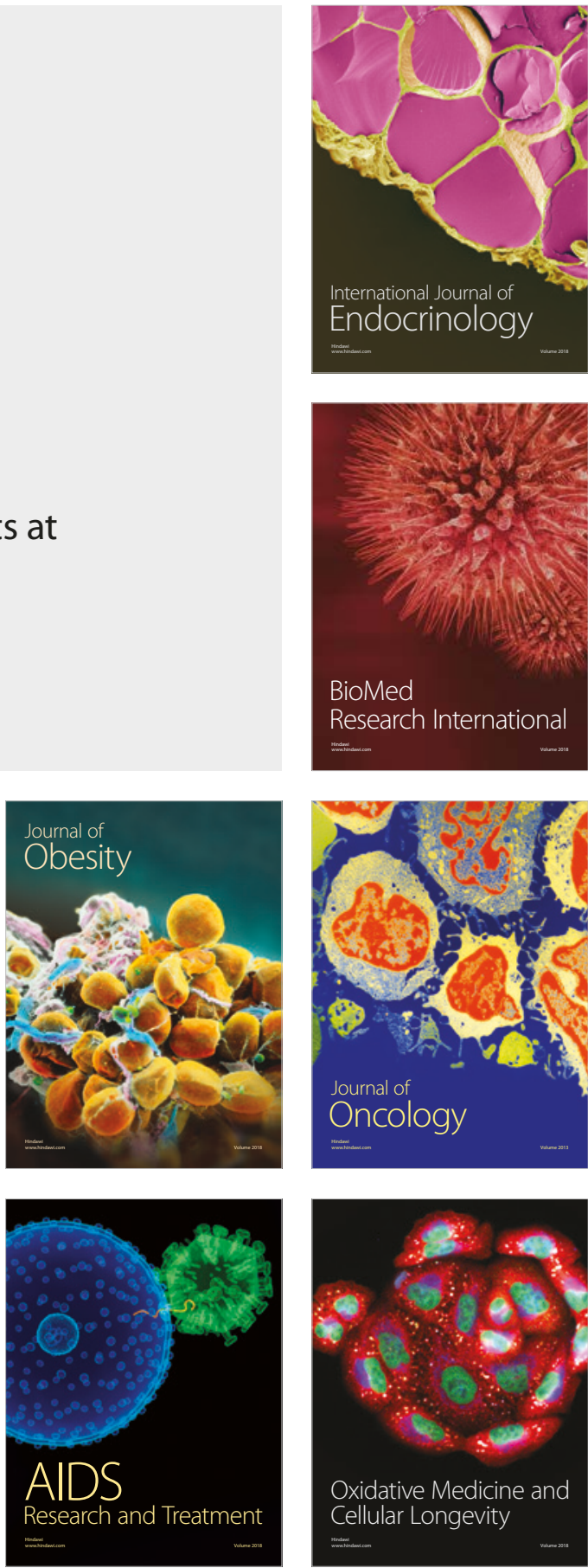